\title{
AIRY-BASED EQUILIBRIUM MESH-FREE METHOD FOR STATIC LIMIT ANALYSIS OF PLANE PROBLEMS
}

\author{
Canh V. Le ${ }^{1, *}$, Phuc L. H. Ho ${ }^{2}$, Hoa T. Nguyen ${ }^{3}$ \\ ${ }^{1}$ International University, Vietnam National University - Ho Chi Minh City, Vietnam \\ ${ }^{2}$ Ho Chi Minh City University of Technology and Education, Vietnam \\ ${ }^{3}$ Ho Chi Minh City Open University, Vietnam \\ *E-mail: lvcanh@hcmiu.edu.vn \\ Received March 16, 2015
}

\begin{abstract}
This paper presents a numerical procedure for lower bound limit analysis of plane problems governed by von Mises yield criterion. The stress fields are calculated based on the Airy function which is approximated using the moving least squares technique. With the use of the Airy-based equilibrium mesh-free method, equilibrium equations are ensured to be automatically satisfied a priori, and the size of the resulting optimization problem is reduced significantly. Various plane strain and plane stress with arbitrary geometries and boundary conditions are examined to illustrate the performance of the proposed procedure.
\end{abstract}

Keywords: Limit analysis, equilibrium mesh-free method, second-order cone programming, Airy function.

\section{INTRODUCTION}

The estimation of the load required to cause collapse of a body or structure plays an important role in design and assessment the safety of many engineering components and structures. If a suitable approximation for the stress field is used, and the static theorem is applied, a lower-bound on the exact limit load can be obtained. In the framework of equilibrium limit analysis formulation, the assumed stress fields are expressed in terms of spatial coordinates and parameters that are usually associated with nodal stress values. These approximated fields are required to satisfy equilibrium conditions over the problem domain. The equilibrium equations are frequently treated in one of two ways in numerical procedures: (i) equilibrium is enforced at nodes in the problem domain and also at boundaries (using the collocation method), or (ii) the equilibrium equations are transformed into the equivalent weak-form (involving integrals), using the so-called weighted residual method. 
Equilibrium finite elements have been developed for static limit analysis problems over the past few decades [1-4]. In [1], a linear-discontinuous element was used in the framework of linear programming. In [2-4], the various triangular finite elements were proposed for use in equilibrium limit analysis formulations for plates and slabs. It has been shown that these constant/linear stress elements can provide good lower bounds on the actual collapse load, but somewhat converge slowly. More recently, equilibrium element-free Galerkin (EFG) mesh-free method based on a moving least squares approximation has been also proposed for lower bound computation of limit load of plates and slabs [5]. The proposed stabilized equilibrium mesh-free method can result in stable and accurate solutions with the use of relatively small number of nodes. In this paper, we will further develop the method by combining the Airy function with the moving least squares approximation. The stress fields will be approximated based on second derivatives of the Airy function, leading to the fact that equilibrium equations will be automatically satisfied a priori. The size of resulting optimization problem is reduced significantly (without equilibrium constraint and only one variable per node).

The layout of the paper is as follows. The next section will describe a static formulation for limit analysis of plane problems. The Airy function and EFG mesh-free method are described in Section 3 and 4, respectively. In section 5, Airy-based equilibrium meshfree formulation for static limit analysis of plane problems will be described. Numerical examples are provided in Section 6 to illustrate the performance of the proposed procedure.

\section{STATIC FORMULATION OF LIMIT ANALYSIS}

Consider a structure covered by a closed area $\Omega$, static boundary $\Gamma_{t}$, kinematic boundary $\Gamma_{u}$, subjected to the external load $t$. The limit analysis formulation is formulated based on assumptions of small deformation and rigid-perfectly plastic material. A static admissible stress field is approximated. Lower bound on the actual collapse load is obtained if equilibrium conditions are satisfied over the problem domain and the yield criterion is not violate every where. The static theorem of limit analysis can be now expressed as

$$
\begin{aligned}
& \lambda=\max \lambda^{-} \\
& \text {s.t } \begin{cases}\nabla \boldsymbol{\sigma}(\mathbf{x})=0, & \forall \mathbf{x} \in \Omega \\
\boldsymbol{n} \boldsymbol{\sigma}(\mathbf{x})=\lambda t, & \forall \mathbf{x} \in \Gamma_{t} \\
\boldsymbol{\psi}[\boldsymbol{\sigma}(\mathbf{x})] \leq 0, & \forall \mathbf{x} \in \Omega\end{cases}
\end{aligned}
$$

where $\psi$ is the yield function. In this study, von Mises failure criterion is used, the yield function is given by

$$
\psi(\sigma)= \begin{cases}\sqrt{\sigma_{x x}^{2}+\sigma_{y y}^{2}+\sigma_{x x} \sigma_{y y}+3 \sigma_{x y}^{2}}-\sigma_{0}, & \text { for plan stress } \\ \sqrt{\frac{1}{4}\left(\sigma_{x x}-\sigma_{y y}\right)^{2}+\sigma_{x y}^{2}}-\sigma_{0}, & \text { for plane strain }\end{cases}
$$

with $\sigma_{x x}, \sigma_{y y}$ and $\sigma_{x y}$ denote the nodal stress components; $\sigma_{0}$ is the yield stress of material. 
The outward surface normal matrix is given as

$$
\boldsymbol{n}=\left[\begin{array}{cc}
n_{x} & 0 \\
0 & n_{y} \\
n_{y} & n_{x}
\end{array}\right]
$$

\section{AIRY STRESS FUNCTION}

Assume the stress function $\varphi(\sigma)$ for a body subjected to only surface load in twodimensions. For plane problems, the stress field has to satisfy the equilibrium equation (with the absent of body loading)

$$
\nabla \boldsymbol{\sigma}(\mathbf{x})=0, \forall \mathbf{x} \in \Omega \text {. }
$$

Eq. (4) can be automatically satisfied if the components of the stress tensor $\sigma$ are calculated by

$$
\sigma_{x x}=\frac{\partial \varphi}{\partial y^{2}}, \quad \sigma_{y y}=\frac{\partial \varphi}{\partial x^{2}}, \quad \sigma_{x y}=-\frac{\partial \varphi}{\partial x \partial y} .
$$

The function $\varphi(\sigma)$, for which the stress components meet Eq. (5), is called Airy stress function. As seen in the equation, when Airy function used, three components of stress tensor can be performed in terms of stress function $\varphi(\sigma)$. Therefore, we need to approximate function $\varphi(\sigma)$ only.

\section{ELEMENT-FREE GALERKIN METHOD [6]}

The approximate function for a set of given scattered nodes in arbitrary domain $\Omega$ using a moving least squares (MLS) technique as follow

$$
u^{h}(\mathbf{x})=\sum_{I=1}^{m} p_{I}\left(\mathbf{x}_{\mathbf{I}}\right) a_{I}(\mathbf{x})=\mathbf{p}^{T}(\mathbf{x}) \mathbf{a}(\mathbf{x}),
$$

where the coefficient vector $\mathbf{a}(\mathbf{x})$ and the basis function vector $\mathbf{p}(\mathbf{x})$ are

$$
\begin{aligned}
& \mathbf{a}(\mathbf{x})=\left[a_{1}(\mathbf{x}), a_{2}(\mathbf{x}), \ldots, a_{m}(\mathbf{x})\right], \\
& \mathbf{p}^{T}(\mathbf{x})=\left[p_{1}(\mathbf{x}), p_{2}(\mathbf{x}), \ldots, p_{m}(\mathbf{x})\right] .
\end{aligned}
$$

The complete polynomial basis is given by

$$
\mathbf{p}^{T}(\mathbf{x})=\left[1, x, y, x y, x^{2}, y^{2}, \ldots, x^{s}, y^{s}\right],
$$

where $s$ is the order of basis function.

The unknown coefficient $\mathbf{a}(\mathbf{x})$ is determined by weighted minimizing the discrete $L_{2}$ norm

$$
J(\mathbf{x})=\sum_{I=1}^{\mathcal{N}} w\left(\mathbf{x}-\mathbf{x}_{I}\right)\left[\mathbf{p}^{T}(\mathbf{x}) \mathbf{a}(\mathbf{x})-u_{I}\right],
$$

where $u_{I}$ represents the nodal value of approximate function at point $I ; \mathcal{N}$ is number of points in the neighborhood of $\mathbf{x} ; w\left(\mathbf{x}-\mathbf{x}_{I}\right)$ is the weight function. In this study, the 
isotropic quartic spline is employed

$$
w\left(\mathbf{x}-\mathbf{x}_{I}\right) \equiv w_{I}(\mathbf{x})= \begin{cases}1-6 s_{I}^{2}+8 s_{I}^{3}-3 s_{I}^{4}, & \text { if } s_{I} \leq 1 \\ 0, & \text { if } s_{I}>1\end{cases}
$$

where $s_{I}=\frac{\left\|\mathbf{x}-\mathbf{x}_{I}\right\|}{R_{I}}$, with $R_{I}$ is the support radius of node $I$.

Minimizing $J(\mathbf{x})$ in Eq. (10) leads to the system of linear equations

$$
\mathbf{A}(\mathbf{x}) \mathbf{a}(\mathbf{x})=\mathbf{B}(\mathbf{x}) \mathbf{u},
$$

with

$$
\begin{aligned}
& \mathbf{u}=\left[u_{1}, u_{2}, \ldots, u_{\mathcal{N}}\right] \\
& \mathbf{A}(\mathbf{x})=\sum_{I=1}^{\mathcal{N}} w\left(\mathbf{x}-\mathbf{x}_{I}\right) \mathbf{p}\left(\mathbf{x}_{I}\right) \mathbf{p}^{T}\left(\mathbf{x}_{I}\right) \\
& \mathbf{B}(\mathbf{x})=\left[w\left(\mathbf{x}-\mathbf{x}_{1}\right) p\left(\mathbf{x}_{1}\right), w\left(\mathbf{x}-\mathbf{x}_{2}\right) p\left(\mathbf{x}_{2}\right), \ldots, w\left(\mathbf{x}-\mathbf{x}_{\mathcal{N}}\right) p\left(\mathbf{x}_{\mathcal{N}}\right)\right] .
\end{aligned}
$$

By inversion, $\mathbf{a}(\mathbf{x})$ can be obtained by solving Eq. (12) as

$$
\mathbf{a}(\mathbf{x})=\mathbf{A}^{-1}(\mathbf{x}) \mathbf{B}_{I}(\mathbf{x}) \mathbf{u} \text {. }
$$

The approximate function can be expressed as

$$
u^{h}(\mathbf{x})=\sum_{I=1}^{\mathcal{N}} \Phi_{I}(\mathbf{x}) u_{I},
$$

where the MLS shape function is defined by

$$
\Phi_{I}(\mathbf{x})=\mathbf{p}^{T}(\mathbf{x}) \mathbf{A}^{-1}\left(\mathbf{x}_{I}\right) \mathbf{B}_{I}(\mathbf{x}) .
$$

Introducing the coefficients $\gamma(\mathbf{x})$ so that

$$
\mathbf{A}(\mathbf{x}) \gamma(\mathbf{x})=\mathbf{p}(\mathbf{x}) \text {. }
$$

The shape function can be rewritten as

$$
\Phi_{I}=\gamma^{T} \mathbf{B}_{I}
$$

Hence, the derivatives of shape function are then given by

$$
\begin{aligned}
& \Phi_{I, j}=\gamma_{, j}^{T} \mathbf{B}_{I}+\gamma^{T} \mathbf{B}_{I, j}, \\
& \Phi_{I, j k}=\gamma_{, j k}^{T} \mathbf{B}_{I}+\gamma_{, j}^{T} \mathbf{B}_{I, k}+\gamma_{, k}^{T} \mathbf{B}_{I, j}+\gamma^{T} \mathbf{B}_{I, j k} .
\end{aligned}
$$

\section{MESH-FREE DISCRETIZATION}

The Airy stress function is approximated for a set of given nodes within the computational domain as

$$
\varphi(\boldsymbol{\sigma})=\sum_{I=1}^{\mathcal{N}} \Phi_{I}(\mathbf{x}) \sigma_{I}
$$

where $\sigma_{I}$ denotes the nodal stress corresponding to nodes $I$. 
The stress components at point $\mathbf{x}$ can be rewritten as

$$
\sigma_{x x}=\sum_{I=1}^{\mathcal{N}} \Phi_{I, y y}(\mathbf{x}) \sigma_{I}, \quad \sigma_{y y}=\sum_{I=1}^{\mathcal{N}} \Phi_{I, x x}(\mathbf{x}) \sigma_{I}, \quad \sigma_{x y}=-\sum_{I=1}^{\mathcal{N}} \Phi_{I, x y}(\mathbf{x}) \sigma_{I} .
$$

As seen in Eq. (24), three stress components $\left(\sigma_{x x}, \sigma_{y y}, \sigma_{x y}\right)$ at an arbitrary node in the domain are now performed via only one variable $\sigma_{I}$. Therefore, problem can be formulated with minimal variables, and reduces the computational cost significantly.

The stress field is now expressed as follows

$$
\boldsymbol{\sigma}^{h}(\mathbf{x})=\left[\begin{array}{l}
\boldsymbol{\sigma}_{x x} \\
\boldsymbol{\sigma}_{y y} \\
\boldsymbol{\sigma}_{x y}
\end{array}\right]=\mathbf{C s},
$$

where

$$
\mathbf{C}=\left[\begin{array}{l}
\mathbf{C}_{x x} \\
\mathbf{C}_{y y} \\
\mathbf{C}_{x y}
\end{array}\right]=\left[\begin{array}{rrrr}
\Phi_{1, y y} & \Phi_{2, y y} & \cdots & \Phi_{\mathcal{N}, y y} \\
\Phi_{1, x x} & \Phi_{2, x x} & \cdots & \Phi_{\mathcal{N}, x x} \\
-\Phi_{1, x y} & -\Phi_{2, x y} & \cdots & -\Phi_{\mathcal{N}, x y}
\end{array}\right]
$$

and

$$
\mathbf{s}^{T}=\left[\sigma_{1}, \sigma_{2}, \cdots \sigma_{\mathcal{N}}\right] .
$$

Problem (1) can be formulated in form of second-order cone programming (SOCP), which can be solved using highly efficient primal-dual interior optimization tools, i.e Mosek [7]. The von Mises criterion is rewritten in terms of a sum of norms as follows

$$
\mathcal{B} \equiv \begin{cases}\mathcal{L}_{P S}=\left\{\boldsymbol{\rho} \in R^{3} \mid \rho_{1} \geq\left\|\rho_{2 \rightarrow 4}\right\|_{L}^{2}=\sqrt{\rho_{2}^{2}+\rho_{3}^{2}+\rho_{4}^{2}}\right\}, & \text { plane stress } \\ \mathcal{L}_{P D}=\left\{\boldsymbol{\rho} \in R^{3} \mid \rho_{1} \geq\left\|\rho_{2 \rightarrow 3}\right\|_{L}^{2}=\sqrt{\rho_{2}^{2}+\rho_{3}^{2}}\right\}, & \text { plane strain }\end{cases}
$$

where $\left(\rho_{1}, \rho_{2}, \rho_{3}, \rho_{4}\right)$ are the additional variables such that

$$
\begin{aligned}
& \rho_{1}=\sigma_{p}, \\
& \rho_{2 \rightarrow 4}=\left[\begin{array}{l}
\rho_{2} \\
\rho_{3} \\
\rho_{4}
\end{array}\right]=\frac{1}{2}\left[\begin{array}{ccc}
2 & 0 & 0 \\
-1 & \sqrt{3} & 0 \\
0 & 0 & 2 \sqrt{3}
\end{array}\right] \text { Cs, plane stress } \\
& \rho_{2 \rightarrow 3}=\left[\begin{array}{l}
\rho_{2} \\
\rho_{3}
\end{array}\right]=\frac{1}{2}\left[\begin{array}{c}
\mathbf{C}_{x x}-\mathbf{C}_{y y} \\
2 \mathbf{C}_{x y}
\end{array}\right] \mathbf{s}, \quad \text { plane strain }
\end{aligned}
$$

The equilibrium conditions are automatically satisfied when Airy stress function is utilized. The lower bound limit analysis can be now formulated as an SOCP optimization problem as

$$
\begin{aligned}
& \lambda=\max \lambda^{-} \\
& \text {s.t } \begin{cases}\mathbf{C} \mathbf{s}=\overline{\mathbf{t}}, & \text { on } \Gamma_{t} \\
\boldsymbol{\rho}^{k} \in \mathcal{L}^{k}, & k=1,2, \ldots, \mathcal{N}_{p}\end{cases}
\end{aligned}
$$

where $\mathcal{N}_{p}$ is number of yield points. 


\section{NUMERICAL EXAMPLES}

Various numerical examples are investigated in order to test the effect of proposed method in this section. von Mises criterion is applied for estimating the plastic limit load of structures. Either plane strain or plane stress problems are considered. The resulting SOCP minimization problems are solved using Mosek (version 6.0) on a $2.50 \mathrm{GHz}$ Intel i5 running Windows 7.

\subsection{Prandtl's punch problem}

The first example deals with a classic plane strain problem, which is the popular benchmark for various numerical approaches. The problem consists a strip footing represented by a semi-infinitive rigid-plastic von Mises medium under a punch load, as shown in Fig. 1(a). Only the right-half domain is modeled due to the symmetry of geometry. The dimensions of computational region are $B=5, H=2$ and the loading is $2 \tau_{0}$. Fig. 1(b) shows the nodal discretization and static boundary conditions.

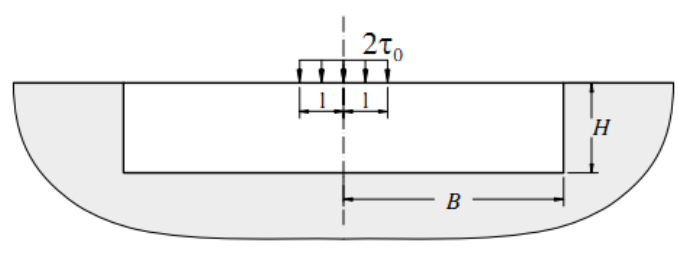

(a) Geometry and loading

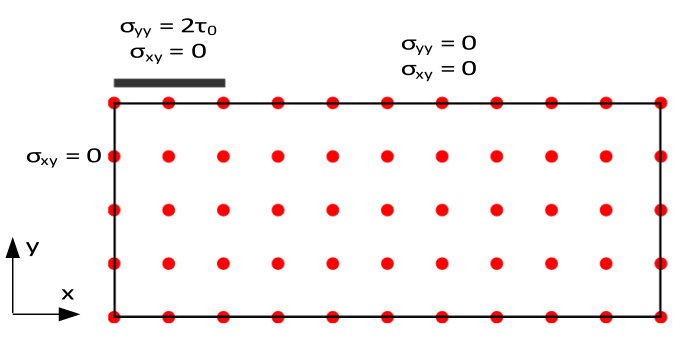

(b) Computational model

Fig. 1. Prandtl's punch problem

The analytical solution obtained by Prandtl is $\lambda=2+\pi=5.142$. Different nodal meshes are considered, the numerical solutions are collected in Tab. 1 and illustrated in Fig. 2. From the table, it can be observed that present results are reasonable, and the lower bound solutions increase corresponding to the rising of the meshes and converge to the analytical value. The relative errors shown in the table also demonstrate the accuracy of proposed method, the error of the best result comparing with Prandtl's solution, with the difference of only $0.03 \%$.

The combination of Airy stress function and SOCP makes the size of the resulting optimization problem reduced significantly. As a result, the optimization problem was solved rapidly, and the optimization CPU time is also summarized in Tab. 1. It is interesting to note that the optimization problem with 5761 variables corresponding to 1440 nodes was handled in only 3 seconds.

The good agreement of proposed method in comparison with previous studies is also shown in Tab. 2. As seen from the table, present result is close to other lower bound solutions and lower than analytical value as well as upper bound solution. This demonstrates the reliability of the approach. 
Table 1. Prandtl's problem: Collapse multiplier

\begin{tabular}{|c|c|c|c|c|c|c|}
\hline $\begin{array}{c}\text { Nodes } \\
\left(\mathcal{N}_{\text {var }}\right)\end{array}$ & 40 & 160 & 360 & 640 & 1000 & 1440 \\
\hline$\lambda^{-}$ & 0.797 & 4.970 & 5.111 & 5.129 & 5.136 & 5.140 \\
$e(\%)$ & 84.49 & 3.34 & 0.61 & 0.25 & 0.12 & 0.03 \\
$t(\mathrm{~s})$ & $<1$ & $<1$ & $<1$ & $<1$ & 2 & 3 \\
\hline
\end{tabular}

$\mathcal{N}_{\text {var }}$ : number of variables in optimization problem

$e$ : relative error

$t$ : CPU-Time

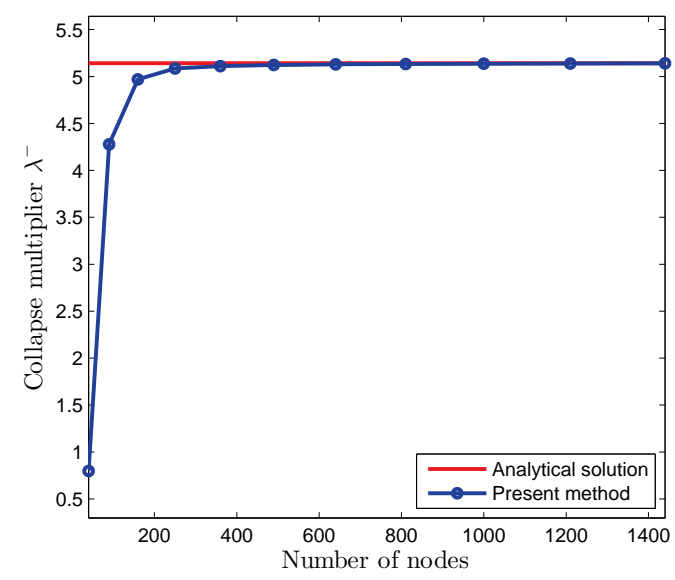

Fig. 2. Prandtl's problem: The collapse multiplier with difference nodal meshes

Table 2. Prandtl's punch problem: Comparison with previous solutions

\begin{tabular}{|l|l|c|}
\hline \multicolumn{1}{|c|}{ Authors } & \multicolumn{1}{|c|}{ Approach } & Collapse multiplier \\
\hline Present method & Static & 5.140 \\
Tin-Loi and Ngo [8] & Static & 5.173 \\
Makrodimopoulos \& Martin [9] & Static & 5.141 \\
\hline Vicente da Silva and Antao [10] & Kinematic & 5.264 \\
Sloan and Kleeman [11] & Kinematic & 5.210 \\
Le et al. [12] & Kinematic & 5.143 \\
Makrodimopoulos and Martin [9] & Kinematic & 5.148 \\
\hline Capsoni and Corradi [13] & Mixed formulation & 5.240 \\
\hline Prandtl [14] & Analytical solution & 5.142 \\
\hline
\end{tabular}




\subsection{Double notched specimen in tension}

This example studies a well-known problem in plane strain as seen in Fig. 3. The given data: $W=L$, the length of crack is taken into account in three cases: $a=L / 3, a=$ $L / 2$ and $a=2 L / 3$. Taking advantage of the symmetry, the upper-right quarter is modeled and the boundary conditions are illustrated as Fig. 3(b).

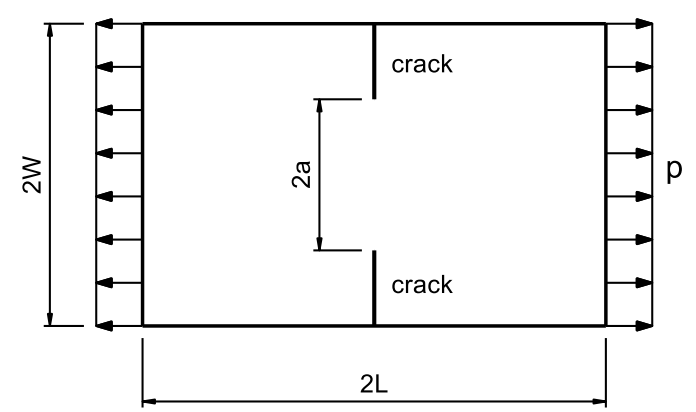

(a) Double notched specimen

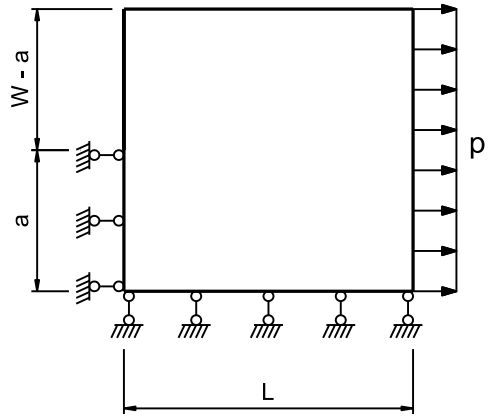

(b) Modeled domain

Fig. 3. Notched specimen subjected to tension

The numerical solutions corresponding to three cases of crack length are shown in Tab. 3. Tab. 4 compares the present solutions with those obtained by other methods. It can be seen that present results are in good agreement with those in the literature.

Table 3. Double notched specimen: Collapse multiplier

\begin{tabular}{|c|c|c|c|c|c|}
\hline \multirow{2}{*}{ Models } & \multicolumn{5}{|c|}{ Number of nodes } \\
\cline { 2 - 6 } & 169 & 361 & 625 & 961 & 1369 \\
\hline$a=L / 3$ & 0.892 & 0.912 & 0.918 & 0.920 & 0.921 \\
$t(\mathrm{~s})$ & $<1$ & $<1$ & $<1$ & 2 & 5 \\
\hline$a=L / 2$ & 1.108 & 1.122 & 1.128 & 1.130 & 1.131 \\
$t(\mathrm{~s})$ & $<1$ & $<1$ & 1 & 2 & 3 \\
\hline$a=2 L / 3$ & 1.350 & 1.372 & 1.378 & 1.380 & 1.381 \\
$t(\mathrm{~s})$ & 1 & 1 & 1 & 4 & 4 \\
\hline
\end{tabular}

t: CPU-Time

In case of $a=L / 2$, the lower bound solutions: $\lambda^{-}=1.131$ and $\lambda^{-}=1.132$ are obtained with 276481 variables for uniform refinement and 83521 variables for adaptive refinement, respectively [15]. In present study, the solution of $\lambda^{-}=1.131$ is obtained with the mesh of 1369 nodes and only 5477 variables in the optimization problem. Furthermore, the time required to handle the problem is also an advantage of this procedure. The solution is obtained rapidly, only about 3 seconds for CPU optimization time. This 
demonstrates proposed method can provide an accurate and reliable result with a low computational cost.

Table 4. Double notched specimen: Comparison with previous solutions

\begin{tabular}{|l|l|c|c|c|}
\hline \multirow{2}{*}{ Authors } & \multicolumn{2}{|c|}{ Approach } & \multicolumn{3}{c|}{ Collapse multiplier } \\
\cline { 3 - 5 } & & $a=L / 3$ & $a=L / 2$ & $a=2 L / 3$ \\
\hline Present method & Static & 0.921 & 1.131 & 1.381 \\
Ciria et al. [15] $^{*}$ & Static & - & 1.131 & - \\
Ciria et al. [15] ** & Static & - & 1.132 & - \\
Krabbenhoft \& Damkilde [16] $^{*}$ & Static & - & 1.132 & - \\
Tin-Loi \& Ngo [8] & Static & $0.947 \ddagger$ & $1.166^{\ddagger}$ & $1.434^{\ddagger}$ \\
\hline Ciria et al. [15] & & - & 1.149 & - \\
Ciria et al. [15] $^{* *}$ & Kinematic & - & 1.139 & - \\
Le et al. [12] & Kinematic & 0.926 & 1.137 & 1.384 \\
\hline Christiansen \& Andersen [17] & Kixed formulation & 0.926 & 1.136 & 1.388 \\
Andersen et al. [18] & Mixed formulation & 0.927 & 1.137 & 1.389 \\
\hline
\end{tabular}

$*$ : uniform refinement

$* *$ : adaptive refinement

‡: where a true lower bound on collapse limit is not guaranteed

\subsection{Thin plates with different cutouts subjected to tension}

A group of square plates with different cutouts in plane stress, consisting square and crack cutout, subjected to tension, as shown in Figs. 4(a) and 4(b), were also considered. One-fourth of plates as shown in Figs. 5(a) and 5(b) are modeled owing to the geometrical symmetry. The computational domains were discretized as shown in Figs. 6(a) and $6(b)$.

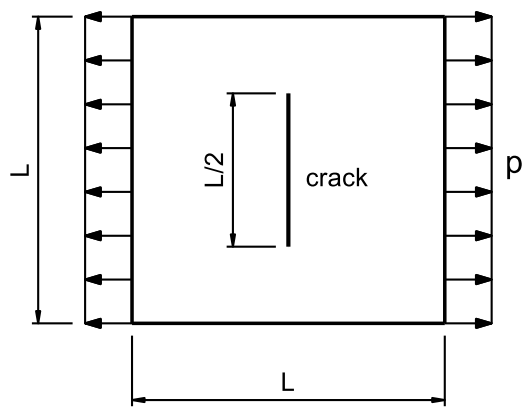

(a) Crack plate

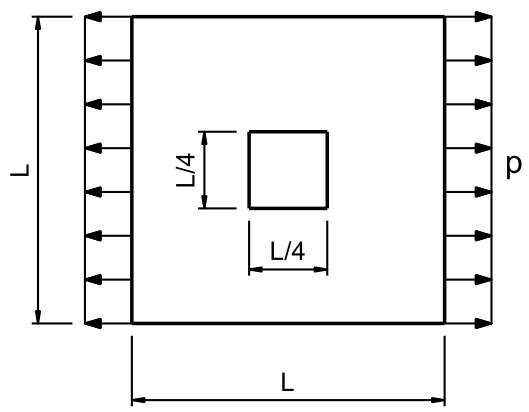

(b) Square cutout plate

Fig. 4. Square plates with central crack and square cutout

Tabs. 5 and 6 report the collapse factors with different nodal distribution. Figs. 7(a) and 7(b) illustrate the convergence rate of solutions. The comparison with other methods 
is described in Tab. 7. From the results, it can be seen that present solutions agree well with those of available approaches in the literature.

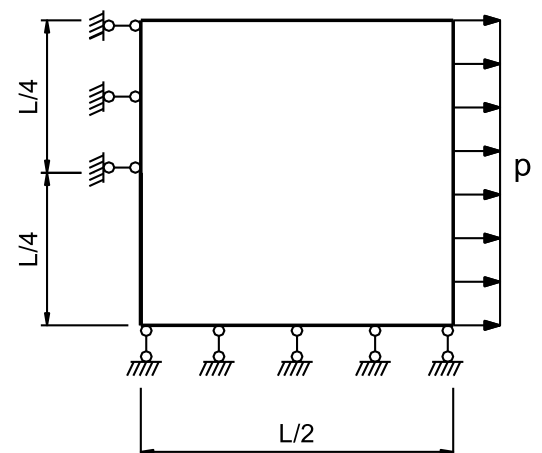

(a) Crack plate

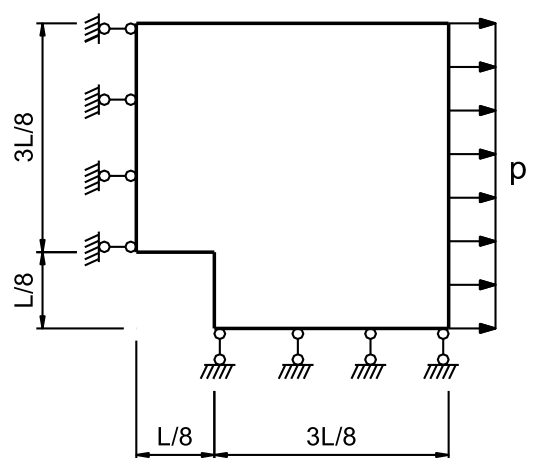

(b) Square cutout plate

Fig. 5. Square plate with central cutout: modeled domains

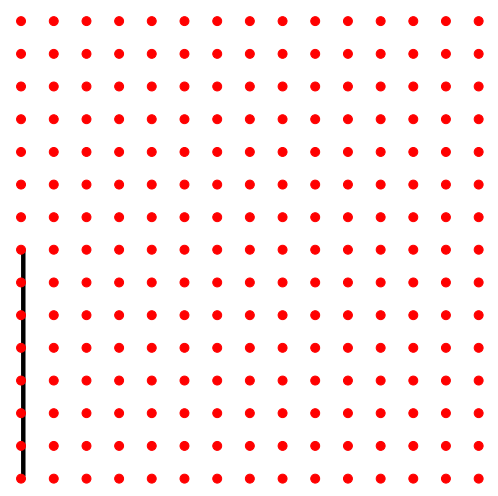

(a) Crack plate

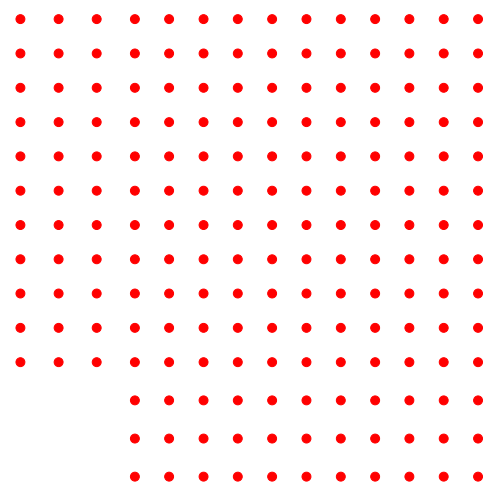

(b) Square cutout plate

Fig. 6. Square plate with central cutout: nodal discretizations

Table 5. Solutions of crack cutout plate problem

\begin{tabular}{|c|c|c|c|c|c|c|}
\hline $\begin{array}{c}\text { Nodes } \\
\left(\mathcal{N}_{\text {var }}\right)\end{array}$ & $\begin{array}{c}42 \\
(246)\end{array}$ & $\begin{array}{c}121 \\
(606)\end{array}$ & $\begin{array}{c}289 \\
(1446)\end{array}$ & $\begin{array}{c}529 \\
(2646)\end{array}$ & $\begin{array}{c}841 \\
(4206)\end{array}$ & $\begin{array}{c}1225 \\
(6126)\end{array}$ \\
\hline$\lambda^{-}$ & 0.210 & 0.451 & 0.481 & 0.491 & 0.495 & 0.498 \\
$t(\mathrm{~s})$ & $<1$ & $<1$ & $<1$ & 1 & 3 & 9 \\
\hline
\end{tabular}

$\mathcal{N}_{\text {var }}:$ number of variables in optimization problem

t: CPU-Time 
Table 6. Solutions of square cutout plate problem

\begin{tabular}{|c|c|c|c|c|c|c|}
\hline $\begin{array}{c}\text { Nodes } \\
\left(\mathcal{N}_{\text {var }}\right)\end{array}$ & $\begin{array}{c}60 \\
(301)\end{array}$ & $\begin{array}{c}160 \\
(801)\end{array}$ & $\begin{array}{c}308 \\
(1541)\end{array}$ & $\begin{array}{c}540 \\
(2701)\end{array}$ & $\begin{array}{c}792 \\
(3961)\end{array}$ & $\begin{array}{c}1288 \\
(6441)\end{array}$ \\
\hline$\lambda^{-}$ & 0.348 & 0.687 & 0.727 & 0.739 & 0.743 & 0.749 \\
$t(\mathrm{~s})$ & $<1$ & $<1$ & $<1$ & 2 & 3 & 5 \\
\hline
\end{tabular}

$\mathcal{N}_{\text {var }}:$ number of variables in optimization problem

$t$ : CPU-Time

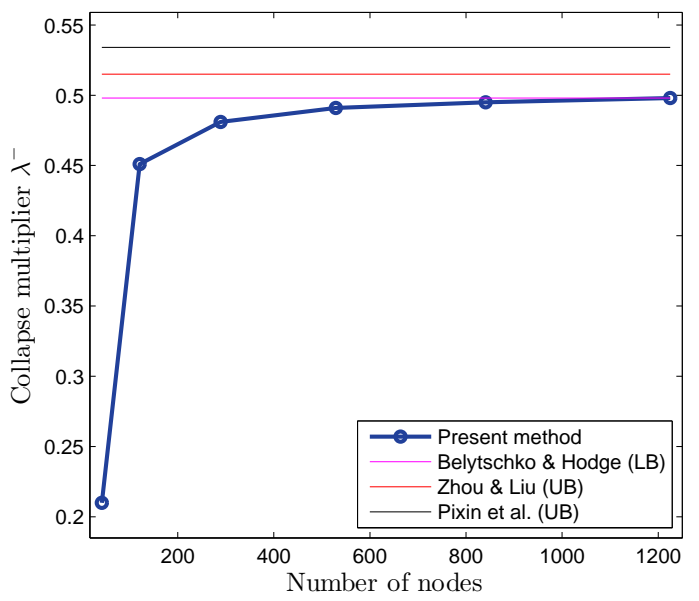

(a) Crack plate

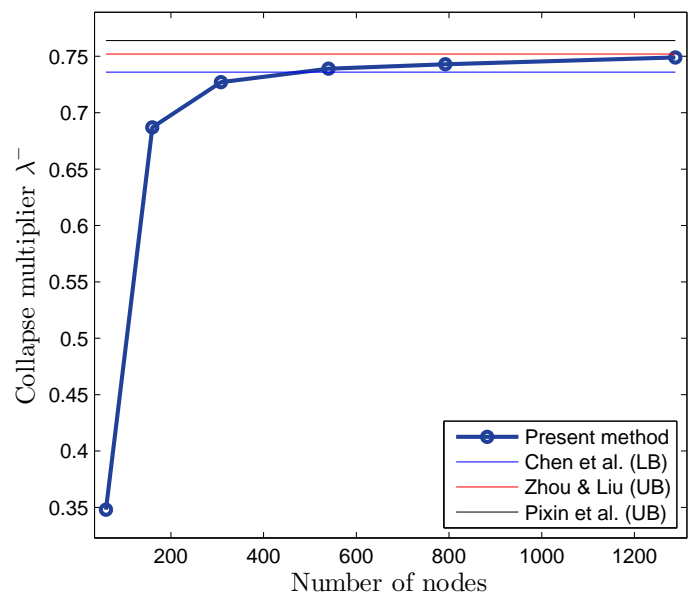

(b) Cutout plate

Fig. 7. Square plate with central cutout: Convergent rate of solutions Table 7. Square plate with central cutout: Comparison with previous solutions

\begin{tabular}{|l|l|c|c|}
\hline \multirow{2}{*}{ Authors } & \multirow{2}{*}{ Approach } & \multicolumn{2}{c|}{ Model } \\
\cline { 3 - 4 } & & Crack plate & Cutout plate \\
\hline Present method & Static & 0.498 & 0.749 \\
Belytschko \& Hodge [19] & Static & 0.498 & 0.693 \\
Chen et al. [20] & Static & 0.513 & 0.736 \\
Zhang et al. [21] & Static & 0.514 & 0.747 \\
\hline Pixin et al. [22] & Kinematic & 0.534 & 0.764 \\
Zhou \& Liu [23] (Sibson) & Kinematic & 0.513 & 0.753 \\
Zhou \& Liu [23] (Laplace) & Kinematic & 0.515 & 0.752 \\
\hline Nguyen-Xuan et al. [24] (NS-FEM-T3) & Dual algorithm & 0.519 & 0.741 \\
Nguyen-Xuan et al. [24] (NS-FEM-T4) & Dual algorithm & 0.530 & 0.747 \\
\hline
\end{tabular}




\section{CONCLUSIONS}

Airy-based equilibrium mesh-free method for lower bound limit analysis of plane strain and plane stress problems has been described. The Airy function is approximated using the moving least squares technique. Stress fields are determined as the second derivatives of the Airy function. Numerical examples were examined to demonstrate that the proposed numerical procedure can provide accurate collapse load using relatively small number of nodes and variables.

\section{ACKNOWLEDGEMENTS}

This research has been supported by the Vietnam National Foundation for Science and Technology Development (NAFOSTED) under grant number 107.02-2013.11.

This research is funded by Vietnam National University Ho Chi Minh City (VNUHCMC) under grant number B2014-28-01.

\section{REFERENCES}

[1] S. Sloan. Lower bound limit analysis using finite elements and linear programming. International Journal for Numerical and Analytical Methods in Geomechanics, 12, (1), (1988), pp. 61-77.

[2] S. Krenk, L. Damkilde, and O. Høyer. Limit analysis and optimal design of plates with equilibrium elements. Journal of Engineering Mechanics, 120, (6), (1994), pp. 1237-1254.

[3] P. N. Poulsen and L. Damkilde. Limit state analysis of reinforced concrete plates subjected to in-plane forces. International Journal of Solids and Structures, 37, (42), (2000), pp. 6011-6029.

[4] K. Krabbenhoft and L. Damkilde. Lower bound limit analysis of slabs with nonlinear yield criteria. Computers \& Structures, 80, (27), (2002), pp. 2043-2057.

[5] C. V. Le, M. Gilbert, and H. Askes. Limit analysis of plates and slabs using a meshless equilibrium formulation. International Journal for Numerical Methods in Engineering, 83, (13), (2010), pp. 1739-1758.

[6] T. Belytschko, Y. Y. Lu, and L. Gu. Element-free Galerkin methods. International Journal for Numerical Methods in Engineering, 37, (2), (1994), pp. 229-256.

[7] A. Mosek. The MOSEK optimization toolbox for MATLAB manual. Version 6.0 Edition, (2011).

[8] F. Tin-Loi and N. S. Ngo. Performance of the p-version finite element method for limit analysis. International Journal of Mechanical Sciences, 45, (6), (2003), pp. 1149-1166.

[9] A. Makrodimopoulos and C. M. Martin. Upper bound limit analysis using simplex strain elements and second-order cone programming. International Journal for Numerical and Analytical Methods in Geomechanics, 31, (6), (2007), pp. 835-865.

[10] A. Capsoni and M. Vicente da Silva. A finite element formulation of Mindlin plates for limit analysis. International Journal for Numerical Methods in Biomedical Engineering, 27, (1), (2011), pp. 143-156.

[11] S. W. Sloan and P. W. Kleeman. Upper bound limit analysis using discontinuous velocity fields. Computer Methods in Applied Mechanics and Engineering, 127, (1), (1995), pp. 293-314.

[12] C. V. Le, H. Nguyen-Xuan, H. Askes, S. Bordas, T. Rabczuk, and H. Nguyen-Vinh. A cellbased smoothed finite element method for kinematic limit analysis. International Journal for Numerical Methods in Engineering, 83, (12), (2010), pp. 1651-1674.

[13] A. Capsoni and L. Corradi. A finite element formulation of the rigid-plastic limit analysis problem. International Journal for Numerical Methods in Engineering, 40, (11), (1997), pp. 20632086. 
[14] L. Prandtl. Über die härte plastischer körper. Nachrichten von der Gesellschaft der Wissenschaften zu Göttingen, Mathematisch-Physikalische Klasse, 1920, (1920), pp. 74-85.

[15] H. Ciria and J. Peraire. Computation of upper and lower bounds in limit analysis using second-order cone programming and mesh adaptivity. In 9th ASCE Specialty Conference on Probabilistic Mechanics and Structural Reliability, (2004), pp. 1-6.

[16] K. Krabbenhoft and L. Damkilde. A general non-linear optimization algorithm for lower bound limit analysis. International Journal for Numerical Methods in Engineering, 56, (2), (2003), pp. 165-184.

[17] E. Christiansen and K. D. Andersen. Computation of collapse states with von mises type yield condition. International Journal for Numerical Methods in Engineering, 46, (8), (1999), pp. 1185-1202.

[18] K. D. Andersen, E. Christiansen, and M. L. Overton. Computing limit loads by minimizing a sum of norms. SIAM Journal on Scientific Computing, 19, (3), (1998), pp. 1046-1062.

[19] T. Belytschko and P. G. J. Hodge. Plane stress limit analysis by finite elements. Journal of the Engineering Mechanics Division, 96, (6), (1970), pp. 931-944.

[20] S. Chen, Y. Liu, and Z. Cen. Lower-bound limit analysis by using the EFG method and nonlinear programming. International Journal for Numerical Methods in Engineering, 74, (3), (2008), pp. 391-415.

[21] X. Zhang, Y. Liu, Y. Zhao, and Z. Cen. Lower bound limit analysis by the symmetric Galerkin boundary element method and the complex method. Computer Methods in Applied Mechanics and Engineering, 191, (17), (2002), pp. 1967-1982.

[22] Z. Pixin, L. Mingwan, and H. Kehchih. A mathematical programming algorithm for limit analysis. Acta Mechanica Sinica, 7, (3), (1991), pp. 267-274.

[23] S.-T. Zhou and Y.-H. Liu. Upper-bound limit analysis based on the natural element method. Acta Mechanica Sinica, 28, (5), (2012), pp. 1398-1415.

[24] H. Nguyen-Xuan, T. Rabczuk, T. Nguyen-Thoi, T. N. Tran, and N. Nguyen-Thanh. Computation of limit and shakedown loads using a node-based smoothed finite element method. International Journal for Numerical Methods in Engineering, 90, (3), (2012), pp. 287-310. 